\title{
Optimal repair of ruptured abdominal aortic aneurysms
}

A ruptured abdominal aortic aneurysm (AAA) is an acute vascular emergency that, if untreated, is nearly always fatal. Ruptured AAAs can now be treated using a novel endovascular aneurysm repair (EVAR) technique, but data from the Immediate Management of Patients with Rupture: Open Versus Endovascular Repair (IMPROVE) trial show that such a strategy is not necessarily superior to open surgery. Long-term follow-up and cost-effectiveness analyses are required before the optimal strategy can be determined.

In the IMPROVE trial, 613 patients with a clinical diagnosis of ruptured AAA were randomly allocated to undergo open surgery or a strategy of CT assessment followed by either EVAR if anatomically suitable or open surgery if not. In those assigned to open repair $(n=297), 261$ patients had a confirmed rupture, 220 underwent open repair, and 36 received EVAR in breach of the trial protocol. In those assigned to the option of EVAR $(n=316), 275$ patients had a confirmed rupture, 174 were anatomically suitable for EVAR, 150 patients underwent the endovascular procedure, and 112 patients underwent open repair (28 in breach of the trial protocol).

The primary end point of the trial (30-day mortality) did not differ significantly between the two groups (35.4\% with the option of EVAR versus $37.4 \%$ with open repair; OR 0.92, 95\% CI 0.66-1.28). Having the option of EVAR seemed to be beneficial in women (30-day mortality $37.1 \%$ versus $57.1 \%$ with open repair; OR 0.44, 95\% CI 0.22-0.91, $P=0.02$ ), but not in men. However, only $22 \%$ of the trial population was female. Overall, no significant differences were observed in $24 \mathrm{~h}$ or in-hospital mortality. Duration of hospital stay was shorter with EVAR, but the additional cost of the endovascular device and consumables meant that the total costs of the two strategies were similar.

Professor Janet Powell, chief investigator in the IMPROVE trial, highlights that the study shows that "endovascular repair can indeed be performed with a low operative mortality (25\%)", compared with $38 \%$ for open repair. However, some patients are not anatomically suitable for EVAR, and others might be switched to open surgery because an endovascular team can take longer to assemble than a team for open repair. The researchers now plan to assess long-term cost-effectiveness and to "identify those patients most suitable for the endovascular approach". They hope that the routine use of EVAR to treat ruptured AAAs will be endorsed by NICE in the UK, and by other regulatory agencies.

Gregory B. Lim

Original article IMPROVE Trial Investigators. Endovascular or open repair strategy for ruptured abdominal aortic aneurysm: 30 day outcomes from IMPROVE randomised trial. BMJ 348, f7661 (2014) 